\title{
Case Report \\ Resection and Abdominal Wall Reconstruction of a Desmoid Tumor with Endometrioma Features
}

\author{
Jaqueline Majors, ${ }^{1}$ Nathaniel F. Stoikes, ${ }^{1}$ Reza Nejati, ${ }^{2}$ and Jeremiah L. Deneve ${ }^{1}$ \\ ${ }^{1}$ Division of Surgical Oncology, Department of Surgery, University of Tennessee Health Science Center, 910 Madison Avenue, Suite 300 , \\ Memphis, TN 38163, USA \\ ${ }^{2}$ Department of Pathology, University of Tennessee Health Science Center, 910 Madison Avenue, Suite 300, Memphis, TN 38163, USA \\ Correspondence should be addressed to Jeremiah L. Deneve; jdeneve@uthsc.edu
}

Received 20 February 2016; Accepted 17 April 2016

Academic Editor: Boris Kirshtein

Copyright (C) 2016 Jaqueline Majors et al. This is an open access article distributed under the Creative Commons Attribution License, which permits unrestricted use, distribution, and reproduction in any medium, provided the original work is properly cited.

\begin{abstract}
Desmoid tumors are rare, musculoaponeurotic mesenchymal origin tumors arising from the proliferation of well-differentiated fibroblasts. Desmoid tumors may arise from any location with the abdominal cavity, abdominal wall and extremity locations being most frequent. We present the case of a 35-year-old female with a history of endometriosis who presented palpable abdominal mass and cyclic abdominal pain. Resection was performed for a presumed desmoid soft tissue tumor. Final pathology demonstrated desmoid histology admixed with abdominal wall endometriosis (endometrioma). This unique pathologic finding has only been rarely reported and is discussed with a brief review of the literature.
\end{abstract}

\section{Case Report}

A 35-year-old female presented with sharp, right lower abdominal pain for several months duration. Her pain was cyclical and worse around her menses. She had a previous surgical history of caesarian section 8 years prior and previous gastric bypass for morbid obesity. On physical examination, a moderate sized mass was present above her Pfannenstiel incision on the right abdominal wall that was firm in nature. Cross-sectional imaging demonstrated a right rectus sheath mass measuring $8 \mathrm{~cm}$, which was well circumscribed and suspicious for a desmoid tumor (Figure 1). There was invasion of the fundus of the uterus (Figure 2). Surgery was recommended.

At the time of exploration, a paramedian incision was created over the area of the abdominal wall mass. The mass involved the right anterior rectus sheath and rectus abdominis muscle. The posterior rectus sheath was largely spared. Inferiorly, near the previous Pfannenstiel incision, the mass was noted to extend into the peritoneum and was adherent to the fundus of the uterus. The mass was resected off the uterus with grossly negative margins. Intraoperative pathologic frozen section demonstrated abdominal wall endometriosis (Figure 3). The abdominal wall defect was too large to allow primary fascial closure. The $11 \times 18 \mathrm{~cm}$ abdominal wall defect was repaired in a complex manner. Large myocutaneous flaps were made and a retrorectus absorbable synthetic mesh underlay was combined with a 30 $\times 30 \mathrm{~cm}$ lightweight and macroporous polypropylene mesh onlay repair. In the subcutaneous space, four 19 French Blake drains were placed. The immediate postoperative course was uncomplicated and she was discharged home at day 4 . After the drains were removed postoperatively, the patient developed an abdominal wall seroma which was managed with percutaneous aspiration without further sequelae. Final pathology revealed an abdominal wall desmoid admixed with florid endometriosis (Figures 4 and 5). She remains without evidence of recurrence at 6-month follow-up.

\section{Discussion}

Desmoid tumors (aggressive fibromatosis) are rare soft tissue tumors that arise from clonal proliferations of mesenchymal stem cells [1]. These tumors derive their name based on their macroscopic appearance from the Greek origin "desmos," 


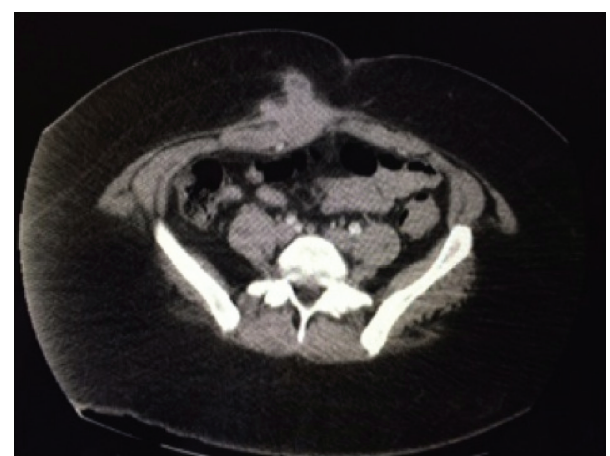

FIGURE 1: Abdominal wall mass arising from the right rectus abdominis.

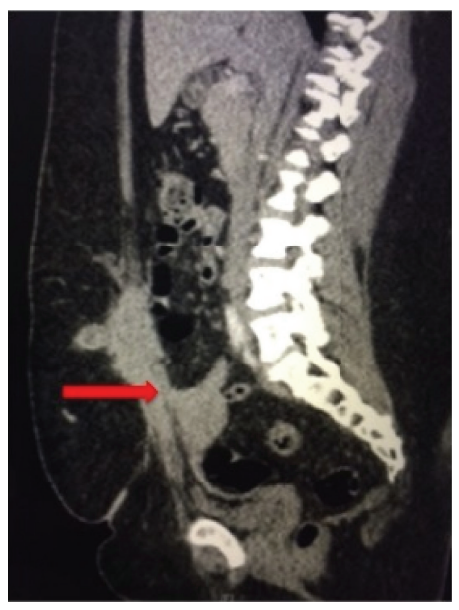

FIgURE 2: Cross-sectional imaging demonstrating invasion of the abdominal wall mass into the uterus (arrow).

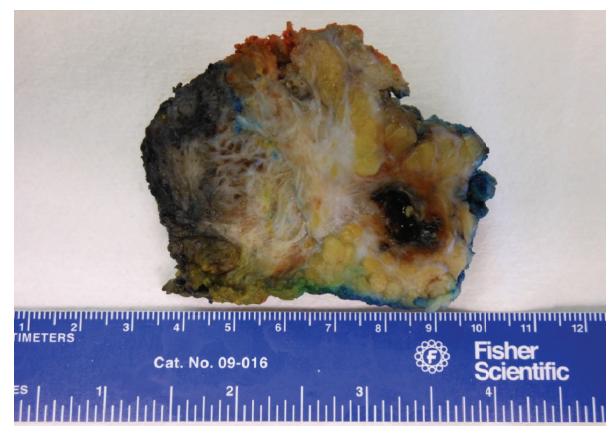

FIGURE 3: Resected abdominal wall mass with desmoid and endometrioma features.

meaning tendon- or band-like. Histologically they are characterized by a fibromatous, benign proliferation of welldifferentiated fibroblast. Desmoid tumors are rare with an estimated incidence of 2-4 cases per million per year and may occur anywhere in the body, with the extremities, abdominal wall, and abdominal cavity being the most common locations [2-4]. A majority of these tumors arise from mutations in the $\beta$-catenin gene, with a smaller proportion associated

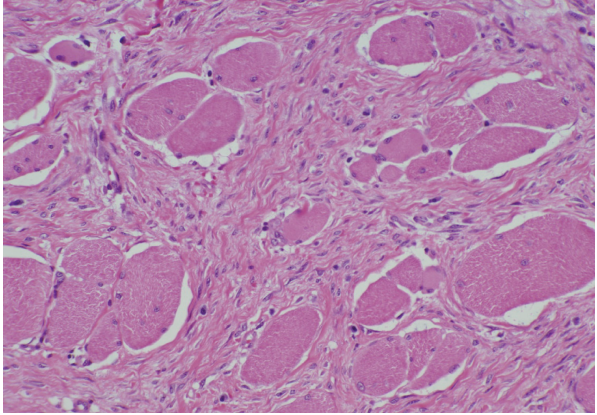

FIGURE 4: Desmoid tumor with irregular infiltration of bland spindle cells between skeletal muscle cells.

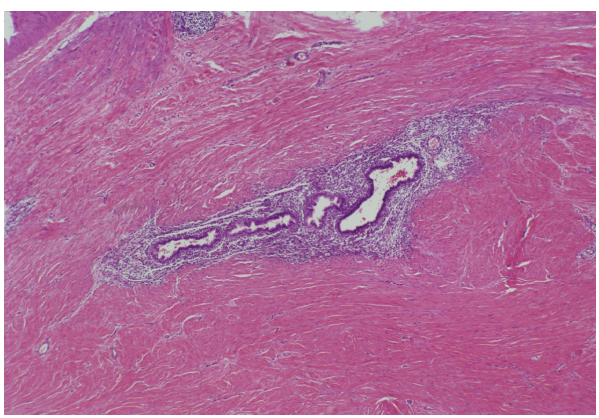

FIGURE 5: Endometriosis with presence of both endometrial glands and stroma.

with mutations in the APC gene and familial adenomatous polyposis $[5,6]$.

While these tumors are considered "benign," the clinical management is highly variable and based on the location and clinical behavior of the primary tumor. In some instances, the lesion remains stable in size and requires no further intervention, while in others the tumors are characterized by rapid growth [7]. Local recurrence rates have been reported as high as $45 \%$ which has led some authors to pursue a conservative "wait-and-see" approach to desmoid tumors [810]. These tumors generally do not metastasize; however they can exhibit aggressive locoregional behavior and invade into adjacent structures.

Surgical resection with negative pathologic margins is the mainstay of therapy for desmoid tumors. Tumor size, location, patient age, and pathologic margin status have all been associated with higher recurrence. Peng and colleagues reviewed the outcome of 211 patients from a multiinstitutional cohort and identified a 53\% 5-year recurrencefree survival [2]. Younger patient age, microscopic-positive margin (R1) status, and extra-abdominal tumor location were all associated with increased recurrence. Reports in the literature, however, have not always been congruent with regard to margin status and an association with recurrence. Gronchi et al. reported the outcome of 203 patients (128, primary, and 75, recurrent) with extra-abdominal aggressive fibromatosis at a single institution [11]. Presenting with recurrent disease was the strongest predictor of local failure. Of those presenting 
with primary desmoid tumors, tumor size and tumor location had prognostic significance while microscopic-positive margin status did not. Postoperative radiation therapy may be considered for those with a positive margin in which relapse or reexcision would be associated with increased morbidity [12]. The role of adjuvant therapy for desmoid tumors has not been standardized. The authors at MD Anderson Cancer Center demonstrated that desmoid tumors are effectively controlled with radiation therapy as an adjunct to surgery when margin-positive resection is anticipated or treating gross disease when surgical resection is not possible [13]. High rates of radiation related complications may develop when treating gross disease; however, this is a prohibitive treatment option for some, especially in situations of truncal or abdominal wall lesions which was the presentation of this patient.

Systemic treatment options exist for locally advanced or unresectable desmoid tumors. Nonsteroidal antiinflammatory drugs (sulindac or celecoxib), hormonal agents (tamoxifen and toremifene), and chemotherapy have all been described to have an efficacy for desmoid tumors [14-18]. Recently, imatinib and sorafenib have both demonstrated promise against desmoid tumors $[19,20]$. Sorafenib use was associated with a partial response in $25 \%$ and stable disease in $70 \%$ of patients when used as first-line therapy or as salvage therapy for those who progress on cytotoxic chemotherapy [20]. Unfortunately, the optimal strategy to manage patients with desmoid tumors has yet to be defined. Crago and colleagues developed a nomogram to better identify candidates who may be ideally suited for surgical management or those who may benefit from neoadjuvant/ systemic therapies and/or nonoperative management [21]. Four hundred ninety-five patients were treated, of which 100 (23\%) recurred. The 5-year local recurrence-free survival was $69 \%$. Abdominal wall tumors had the best overall outcome, with a long-term disease-free survival of over $90 \%$. Tumor size, tumors located on the extremities, and patient age were all associated with increased risk of recurrence, while margin status had no impact. The authors concluded that a postoperative nomogram including age, tumor size, and location predicts recurrence and aids in decision-making. They further suggested that systemic therapy might be appropriate for young patients with large extremity tumors while surgery alone may be sufficient for abdominal wall lesions.

Endometriosis is defined as the presence of ectopic endometrial functional glands and stroma. Abdominal wall endometriosis presenting as a mass (endometrioma) has been documented in the scar of prior abdominal incisions or more commonly after caesarian section and is estimated to occur in as many as up to $3.5 \%$ of patients with endometriosis after surgical treatment $[22,23]$. The likely pathogenesis is related to direct implantation of endometrial tissue during an abdominal surgical procedure. Women with endometriomas can present with a variety of complaints, notably a palpable abdominal mass or cyclic abdominal pain related to menses [24]. Surgical resection is the primary treatment modality for endometriomas. Reconstruction after resection of larger lesions may require mesh reinforcement [25].
Medical therapy with oral contraceptives is largely ineffective [26]. In the situation of the case report described above, we chose to reconstruct the abdominal wall using a complex retrorectus mesh underlay technique with onlay mesh reinforcement with fibrin glue. We have performed this fixation approach routinely at our institution and found it to be less technically demanding with shorter operative times than traditional transfascial repair and less long-term pain [27]. Resection of endometriomas is warranted as there has been an association with other malignancies, such as clear cell adenocarcinoma [28]. Indeed, few isolated reports of abdominal wall endometriosis and desmoid tumor pathology have also been reported [29-31]. Estrogen exposure has also been associated as a risk factor for desmoid tumor formation [32]. The etiology, association, and long-term outcome of desmoid tumor with endometriosis features are currently unknown. At present, the patient presented in this brief report is greater than 6 months without tumor recurrence. Long-term followup and multi-institutional reviews are required to better understand the natural history of this rare phenomenon.

\section{Conclusion}

Desmoid tumors are rare, soft tissue tumors arising from mesenchymal origin. Tumors arising from the abdominal wall tend to have a better long-term outcome than those arising from the extremity. Surgery remains the mainstay of therapy with radiation therapy or systemic therapy reserved for those who are unresectable at presentation or in which resection would confer significant function-limiting morbidity. The association of endometriosis features with desmoid tumors is rare. Surgical resection and close follow-up are required for both tumor types. The long-term outcome for this tumor association remains to be determined.

\section{Disclosure}

This study is presented in part at the 2014 Tennessee Chapter of the American College of Surgeons Annual Meeting, Paris Landing, TN.

\section{Competing Interests}

The authors declare that they have no competing interests.

\section{References}

[1] C. Wu, S. Nik-Amini, P. Nadesan, W. L. Stanford, and B. A. Alman, "Aggressive fibromatosis (desmoid tumor) is derived from mesenchymal progenitor cells," Cancer Research, vol. 70, no. 19, pp. 7690-7698, 2010.

[2] P. D. Peng, O. Hyder, M. N. Mavros et al., "Management and recurrence patterns of desmoids tumors: a multi-institutional analysis of 211 patients," Annals of Surgical Oncology, vol. 19, no. 13, pp. 4036-4042, 2012.

[3] J. J. Reitamo, T. M. Schelnin, and P. Häyry, “The desmoid syndrome. New aspects in the cause, pathogenesis and treatment of the desmoid tumor," The American Journal of Surgery, vol. 151, no. 2, pp. 230-237, 1986. 
[4] S. R. Phillips, R. A'Hern, and J. M. Thomas, "Aggressive fibromatosis of the abdominal wall, limbs and limb girdles," British Journal of Surgery, vol. 91, no. 12, pp. 1624-1629, 2004.

[5] S. Tejpar, F. Nollet, C. Li et al., "Predominance of beta-catenin mutations and beta-catenin dysregulation in sporadic aggressive fibromatosis (desmoid tumor)," Oncogene, vol. 18, no. 47, pp. 6615-6620, 1999.

[6] E. Robanus-Maandag, C. Bosch, S. Amini-Nik et al., "Familial adenomatous polyposis-associated desmoids display significantly more genetic changes than sporadic desmoids," PLoS ONE, vol. 6, no. 9, Article ID e24354, 2011.

[7] J. J. Lewis, P. J. Boland, D. H. Y. Leung, J. M. Woodruff, and M. F. Brennan, "The enigma of desmoid tumors," Annals of Surgery, vol. 229, no. 6, pp. 866-872, 1999.

[8] K. Huang, H. Fu, Y.-Q. Shi, Y. Zhou, and C.-Y. Du, "Prognostic factors for extra-abdominal and abdominal wall desmoids: a 20-year experience at a single institution," Journal of Surgical Oncology, vol. 100, no. 7, pp. 563-569, 2009.

[9] M. T. Ballo, G. K. Zagars, A. Pollack, P. W. T. Pisters, and R. A. Pollock, "Desmoid tumor: prognostic factors and outcome after surgery, radiation therapy, or combined surgery and radiation therapy," Journal of Clinical Oncology, vol. 17, no. 1, pp. 158-167, 1999.

[10] M. Fiore, F. Rimareix, L. Mariani et al., "Desmoid-type fibromatosis: a front-line conservative approach to select patients for surgical treatment," Annals of Surgical Oncology, vol. 16, no. 9, pp. 2587-2593, 2009.

[11] A. Gronchi, P. G. Casali, L. Mariani et al., "Quality of surgery and outcome in extra-abdominal aggressive fibromatosis: a series of patients surgically treated at a single institution," Journal of Clinical Oncology, vol. 21, no. 7, pp. 1390-1397, 2003.

[12] M. von Mehren, R. S. Benjamin, M. M. Bui et al., "Soft tissue sarcoma, version 2.2012: featured updates to the NCCN guidelines," Journal of the National Comprehensive Cancer Network, vol. 10, no. 8, pp. 951-960, 2012.

[13] M. T. Ballo, G. K. Zagars, P. Pisters, and A. Pollack, “The role of radiation therapy in the management of dermatofibrosarcoma protuberans," International Journal of Radiation Oncology Biology Physics, vol. 40, no. 4, pp. 823-827, 1998.

[14] J. Janinis, M. Patriki, L. Vini, G. Aravantinos, and J. S. Whelan, "The pharmacological treatment of aggressive fibromatosis: a systematic review," Annals of Oncology, vol. 14, no. 2, pp. 181190, 2003.

[15] V. P. de Camargo, M. L. Keohan, D. R. D’Adamo et al., "Clinical outcomes of systemic therapy for patients with deep fibromatosis (desmoid tumor)," Cancer, vol. 116, no. 9, pp. 2258-2265, 2010.

[16] A. Hansmann, C. Adolph, T. Vogel, A. Unger, and G. Moeslein, "High-dose tamoxifen and sulindac as first-line treatment for desmoid tumors," Cancer, vol. 100, no. 3, pp. 612-620, 2004.

[17] P. Maseelall, J. C. Robins, D. B. Williams, and M. A. Thomas, "Stabilization and regression of a recurrent desmoid tumor with the antiestrogen toremifene," Fertility and Sterility, vol. 84, no. 2, pp. 509.e11-509.e13, 2005.

[18] D. Garbay, A. Le Cesne, N. Penel et al., "Chemotherapy in patients with desmoid tumors: a study from the French Sarcoma Group (FSG)," Annals of Oncology, vol. 23, no. 1, Article ID mdr051, pp. 182-186, 2012.

[19] N. Penel, A. Le Cesne, B. N. Bui et al., "Imatinib for progressive and recurrent aggressive fibromatosis (desmoid tumors): an
FNCLCC/French Sarcoma Group phase II trial with a longterm follow-up," Annals of Oncology, vol. 22, no. 2, pp. 452-457, 2011.

[20] M. M. Gounder, R. A. Lefkowitz, M. L. Keohan et al., "Activity of sorafenib against desmoid tumor/deep fibromatosis," Clinical Cancer Research, vol. 17, no. 12, pp. 4082-4090, 2011.

[21] A. M. Crago, B. Denton, S. Salas et al., "A prognostic nomogram for prediction of recurrence in desmoid fibromatosis," Annals of Surgery, vol. 258, no. 2, pp. 347-353, 2013.

[22] J. Kang, J.-H. Baek, W.-S. Lee et al., "Clinical manifestations of abdominal wall endometriosis: a single center experience," Archives of Gynecology and Obstetrics, vol. 287, no. 2, pp. 301305, 2013.

[23] G. M. Honoré, "Extrapelvic endometriosis," Clinical Obstetrics and Gynecology, vol. 42, no. 3, pp. 699-711, 1999.

[24] G. K. Patterson and G. B. Winburn, "Abdominal wall endometriomas: report of eight cases," American Surgeon, vol. 65, no. 1, pp. 36-39, 1999.

[25] A. M. Ecker, N. M. Donnellan, J. P. Shepherd, and T. T. M. Lee, "Abdominal wall endometriosis: 12 years of experience at a large academic institution," American Journal of Obstetrics and Gynecology, vol. 211, no. 4, pp. 363.el-363.e5, 2014.

[26] S. K. Chatterjee, "Scar endometriosis: a clinicopathologic study of 17 cases," Obstetrics and Gynecology, vol. 56, no. 1, pp. 81-84, 1980.

[27] N. Stoikes, D. Webb, B. Powell, and G. Voeller, "Preliminary report of a sutureless onlay technique for incisional hernia repair using fibrin glue alone for mesh fixation," The American Surgeon, vol. 79, no. 11, pp. 1177-1180, 2013.

[28] H. Liu, J. Leng, J. Lang, and Q. Cui, "Clear cell carcinoma arising from abdominal wall endometriosis: a unique case with bladder and lymph node metastasis," World Journal of Surgical Oncology, vol. 12, article 51, 2014.

[29] M. G. Muto, M. J. O’Neill, and E. Oliva, "Case 18-2005: a 45year-old woman with a painful mass in the abdomen," The New England Journal of Medicine, vol. 352, no. 24, pp. 2535-2542, 2005.

[30] H. Krentel, G. Tchartchian, and R. L. De Wilde, "Desmoid tumor of the anterior abdominal wall in female patients: comparison with endometriosis," Case Reports in Medicine, vol. 2012, Article ID 725498, 4 pages, 2012.

[31] M. Koshariya, S. Shukla, Z. Khan et al., "Giant desmoid tumor of the anterior abdominal wall in a young female: a case report," Case Reports in Surgery, vol. 2013, Article ID 780862, 4 pages, 2013.

[32] M. N. Kulaylat, C. P. Karakousis, C. M. Keaney, D. McCorvey, J. Bem, and J. L. Ambrus Sr., "Desmoid tumour: a pleomorphic lesion," European Journal of Surgical Oncology, vol. 25, no. 5, pp. 487-497, 1999. 


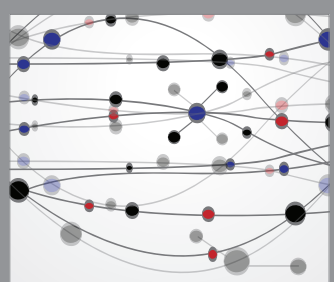

The Scientific World Journal
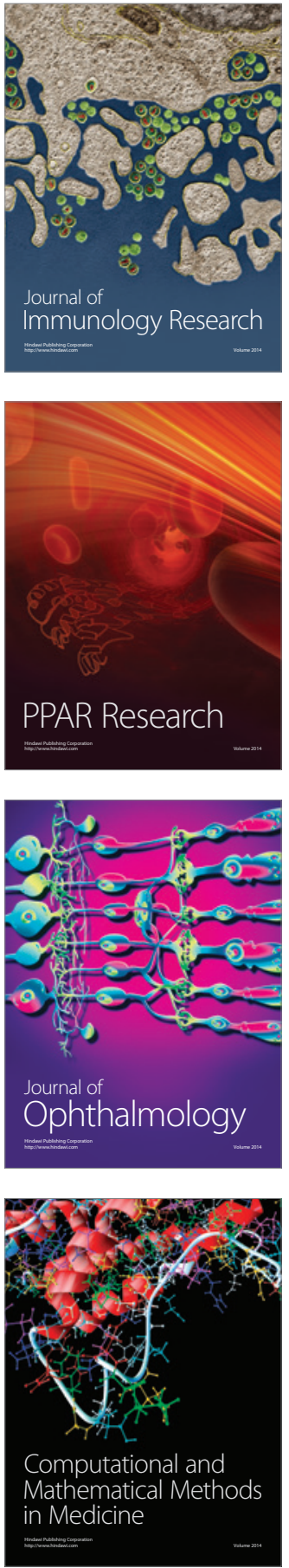

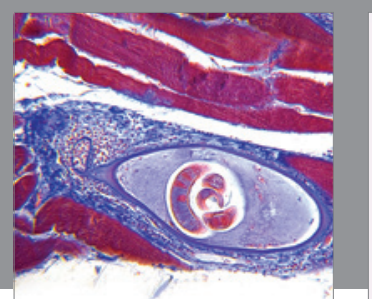

Gastroenterology Research and Practice

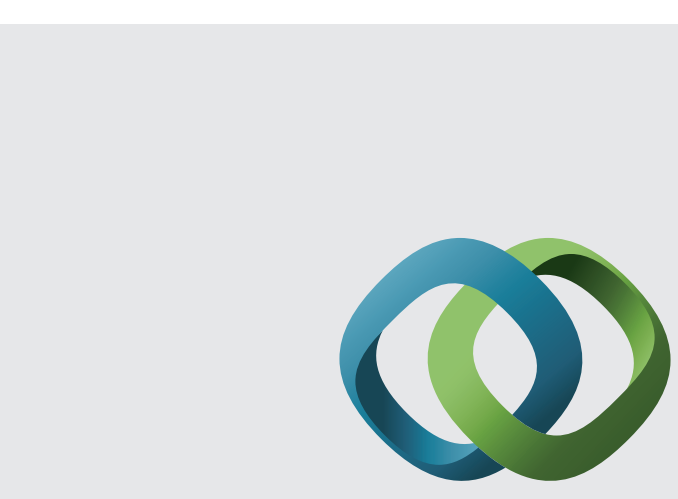

\section{Hindawi}

Submit your manuscripts at

http://www.hindawi.com
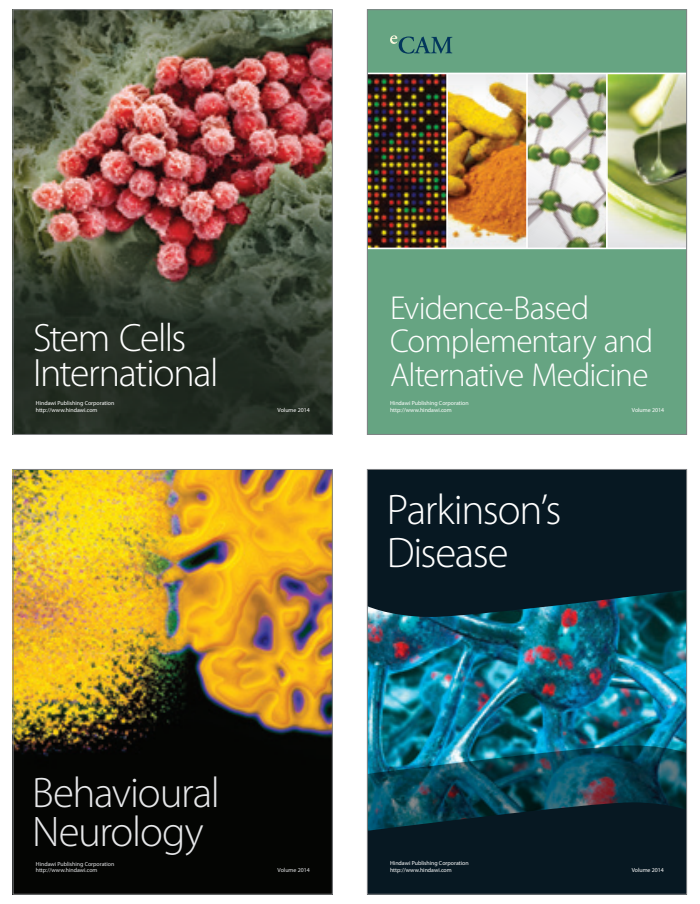
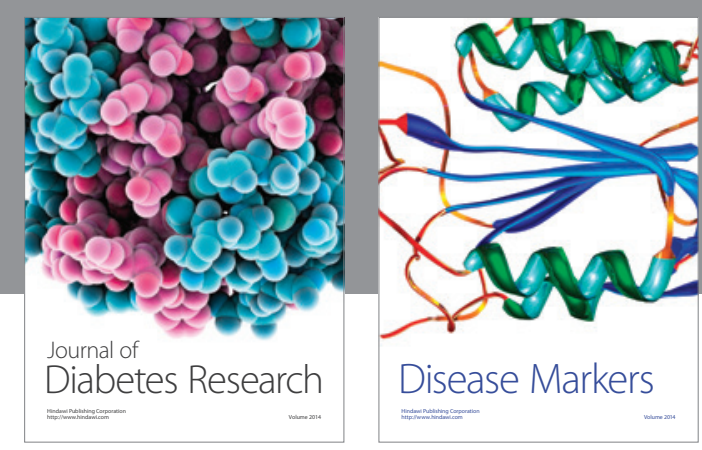

Disease Markers
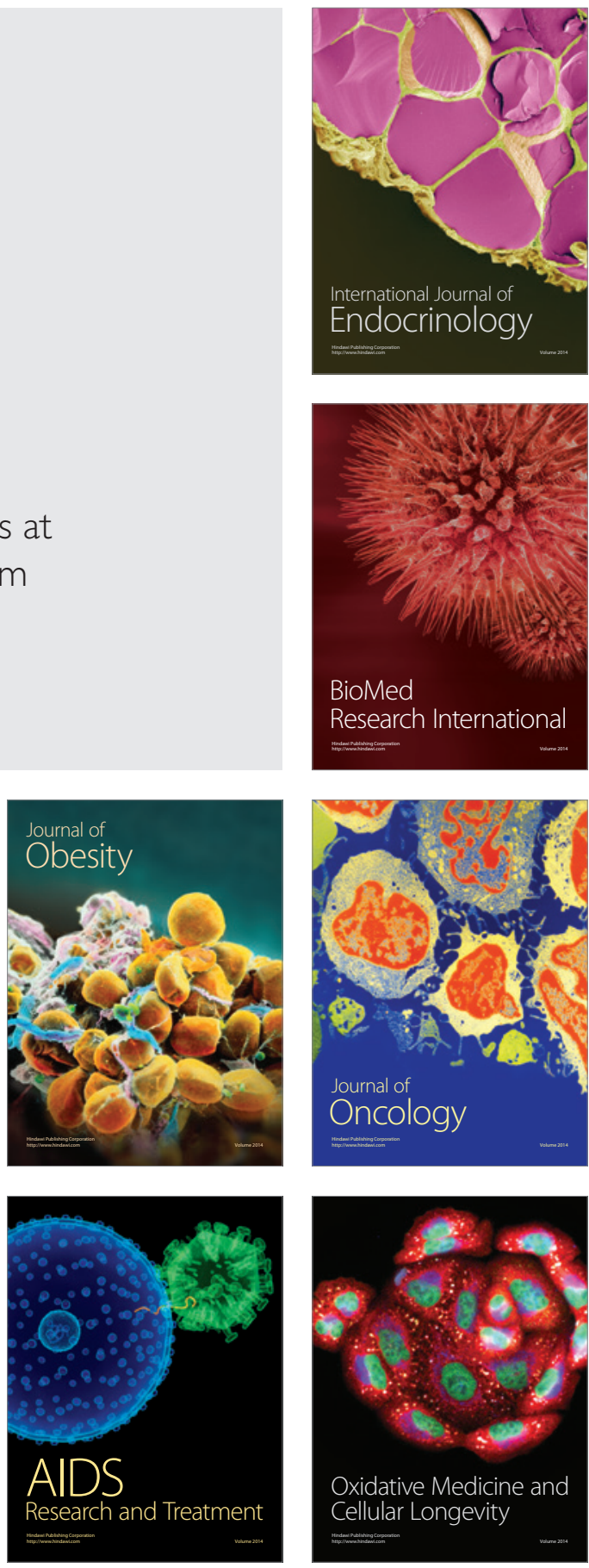